Caddy of King's College Hospital for the help he rendered me throughout the case by his watching and careful attention to the patient during his illness.

Earl's-court-gardens, S.W.

\section{CASE OF RASH RESEMBLING SCARLET FEVER.}

\section{By Lours E. Stefyenson, M.B., B.C. Cantab.}

ON Oct. 18th, 1894 I was called to see a child said to be suffering from scarlet fever. Neither pyrexia nor angina was present. There was a rash covering the neck, the chest, most of the back, and the abdomen down to the level of two inches or so below the umbilicus. It had a remarkable resemblance to the rash of scarlet fever, showing a uniform pink ground dotted thickly over with scarlet points. At the apper and lower edges of the eruption there were isolated scarlet points. There was no rash anywhere else. The limited area of rash and its almost sharply defined upper and lower edges were suspicious, and I asked the mother if the child was wearing new flannel. She said not, but she informed me that she had washed the child's woollen undervest with water to which some paraffin oil had been added, and on examination the garment in question had a strong odour of that oil. The rash quickly faded away, there was no desquamation, and although there were two or three more children in the house who had never had scarlet fever, no more cases of rash occurred. The house was also a very insanitary one, so that I think there can be little doubt that this was a case of artificial rash caused by irritation from paraffin oil.

Temple Sowerby, Penrith.

CASE OF PURPURA HAMORRHAGICA FOLLOWING "MALTA FEVER" OR "MEDITERRANEAN FEVER."

BY G. E. Macheod, L.R.C.P. \& S. EdiN., L.F.P.S. GLasG. LATE ASSISTANT PHYSICIAN, DISTRICT ASYLUM, INVERNESS.

THE following account of a rapidly fatal case of purpura hæmorrhagica occurring during an early period of convalescence from an acute attack of "Malta fever" or "Mediterranean fever," which came under my notice a few months ago, may be of interest to some of the readers of THE LANCET. The patient, a naval officer, while stationed at Malta, contracted a sharp attack of so-called "Mediterranean fever," for which he was under treatment on the island for about seven weeks, being invalided home at a very early period of convalescence by his medical attendants. When I first saw him he was in an extremely emaciated and very weak condition, having to be carried on board the steamer, and being quite unable to raise himself from the horizontal position without assistance. $\mathrm{He}$ spoke with difficulty and seemed to be in great mental distress, constantly brooding over his troubles, and taking a very gloomy view of his condition. His face was pale and exsanguine, the tongue was thickly coated and tremulous, and the bowels were obstinately constipated. The radial pulse was very weak and rapid and the temperature was slightly subnormal. On his arrival on board he was immediately given a little stimulant and placed on a nutritious liquid diet of beef tea and milk, administered alternately at short intervals, and a mixture containing iron and arsenic was prescribed, the bowels being freely opened by enema. Under this treatment the patient improved considerably, and when seen early the next morning was found to be much brighter and more cheerful, having had a good night's rest. The pulse was now much stronger and less rapid and the temperature normal $\left(98.4^{\circ} \mathrm{F}\right.$.). His face was less anæmic in appearance and his hands less tremulous, and he seemed to take much more interest in his surroundings, remarking how much better he felt, and that he thought the voyage was already doing him good. This improvement continued for two days, during which time there were no particular symptoms needing attention, the patient going on satisfactorily. On the morning of the third day, however, it was noticed for the first time that several small purpuric spots had made their appearance on his legs and forearms, and although he did not complain of feeling any worse he was decidedly weaker and less cheerful than on the previous day.
The temperature was $99^{\circ}$ and the pulse 80 . Towards evening the patient bad a slight attack of epistaxis, followed in about three hours' time by a much more severe one, the blood escaping freely in spite of the usual milder treatment and finally necessitating plugging of the anterior nares which succeeded in stopping it for a time. (The posterior nares were also carefully examined, but no blood could be seen escaping through them.) Shortly after this : general sanguineous oozing commenced from the mucous lining of the month, the blood collecting round the bases of the teeth and then being swallowed and vomited, thus simulating hæmatemesis. The patient was kept absolutel at rest in the horizontal position in a cool room, and liquid extract of ergot was administered in thirty-minim doses, astringent mouth washes being used frequently, which, however, seemed to have merely a temporary styptic effect. As there was no hæmatemesis excepting the spurious form of it above alluded to, liquid nourishment was still given by the stomach in small quantities and iced as a precantionary measure. An interval of about two hours now elapsed during which there was practically no bleeding at all, but after this it recommenced with redoubled severity, hæmaturia being now noticed for the first time, the blood (which was of a bright red colour) being passed towards the end of micturition, pointing to vesical hxmorrhage. Ergotin was now injected hypodermically and other astringents freely administered, but in spite of all treatment the patient rapidly grew weaker and weaker, passing into an extremely anæmic state, and finally sinking from loss of blood and exhaustion. The exceptionally malignant form of the disease as exemplified by this case makes it not devoid of interest, resembling as it does so closely a rapidly fatal type of hæmorrhagic purpura (purpura fulminans) alluded to by Roberts in the ninth edition of his "Theory and Practice of Medicine." The pathology of the purpura, which occurs during convalescence from fever, is interesting; thus Immermann, as quoted by Fagge, in the third edition of his "Text.book on the Principles and Practice of. Medicine," suggests that "it is possibly due to the circumstance that the recovery of the minute vessels is sometimes retarded beyond the time at which the volnme of the blood is restored and the heart regains its vigour." It would be extremely interesting to know in this connexion whether the defiaite micrococcus which, according to Dr. David Bruce, is to be found in the organs in every fatal case of "Malta fever" is capable of producing those changes in the blood and small vessels to which morbus maculosus is generally attributed.

Guilford-street, W.C.

\section{ARREST OF DEVELOPMENT OF THE CRANIAL BONES} By Henry George Dixon, M.R.C.S. Eng.,

$$
\text { L.R.C.P. IREL. }
$$

ON the evening of June 30 th, 1895, a primipara was delivered without assistance of a seven months' female child, who was found dead in bed attached to the placenta, which was in the vagina. The trunk and lower extremities presented no peculiarites. There appeared to be no neck, the absence of several cervical vertebræ giving the face the appearance of being sessile on the thorax. The eyes, which were very prominent, had the appearance of being placed on the summit of the cranium, which sloped away backwards immediately behind them. There were no upper orbital plates, the frontal bone being absent, as were also both parietals and the greater part of the occipital and temporal bones. At the back of the eyes and nose was a bony mass requiring the use of the saw to divide it ; it was about three-quarters of an inch thick and contained a small cavity in which there was about half a drachm of dark-red matter of the consistence of marrow. It appeared as though the basilar processes of the occipital and sphenoid and the petrous portions of both temporals bad coalesced and grown together. The spinal canal was patulous at its upper extremity; there the skin ceased, the bony mass being covered by a dark-red grumous substance. Both lungs floated in water. The mother asserted that the child, whose length was twelve inches and three-quarters and weight one and three-quarter pounds, was stillborn. I presume that the development of the bones forming the vault of the cranium did not advance beyond the membranous stage, and that the membrane gave way during birth

Gibson-square, $\mathrm{N}$, 